\title{
Plant genera Cannabis and Humulus share the same pair of well- differentiated sex chromosomes
}

\section{Djivan Prentout $^{1}$ (D), Natasa Stajner ${ }^{2}$, Andreja Cerenak ${ }^{3}$, Theo Tricou ${ }^{1}$ (D), Celine Brochier-Armanet ${ }^{1}$, Jernej Jakse $^{2}$ (D) Jos Käfer ${ }^{{ }^{*}}$ (D) and Gabriel A. B. Marais ${ }^{1,4^{*}}$ (D)}

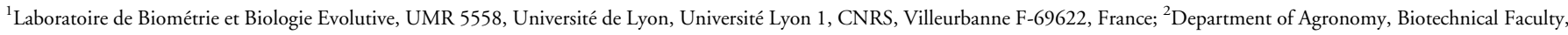

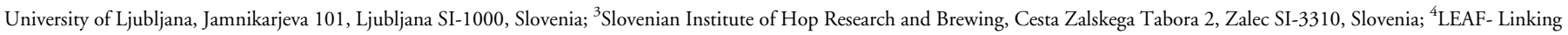
Landscape, Environment, Agriculture and Food, Instituto Superior de Agronomia, Universidade de Lisboa, Lisboa 1349-017, Portugal

Author for correspondence: Diivan Prentout

Email:djivan.prentout@univ-lyon1.fr

Received: 19 February 2021

Accepted: 29 April 2021

New Phytologist (2021)

doi: $10.1111 /$ nph.17456

Key words: Cannabaceae, dioecy, dosage compensation, Humulus lupulus, sex chromosomes, Y degeneration.

\section{Summary}

- We recently described, in Cannabis sativa, the oldest sex chromosome system documented so far in plants (12-28 Myr old). Based on the estimated age, we predicted that it should be shared by its sister genus Humulus, which is known also to possess XY chromosomes.

- Here, we used transcriptome sequencing of an $\mathrm{F}_{1}$ family of $H$. lupulus to identify and study the sex chromosomes in this species using the probabilistic method SEX-DETECTOR.

- We identified 265 sex-linked genes in $H$. lupulus, which preferentially mapped to the $C$. sativa $X$ chromosome. Using phylogenies of sex-linked genes, we showed that a region of the sex chromosomes had already stopped recombining in an ancestor of both species. Furthermore, as in $C$. sativa, $Y$-linked gene expression reduction is correlated to the position on the $X$ chromosome, and highly $Y$ degenerated genes showed dosage compensation.

- We report, for the first time in Angiosperms, a sex chromosome system that is shared by two different genera. Thus, recombination suppression started at least 21-25 Myr ago, and then (either gradually or step-wise) spread to a large part of the sex chromosomes (c. $70 \%$ ), leading to a degenerated $Y$ chromosome.

\section{Introduction}

Among $>15000$ dioecious angiosperm species (i.e. species with separate sexes; Renner, 2014), < 20 sex chromosome systems have been studied with genomic data (Ming et al., 2011; Baránková et al., 2020). Most plants with sex chromosomes exhibit male heterogamety, with XY chromosomes in males and XX chromosomes in females (Westergaard, 1958; Charlesworth, 2016). The portion of the $Y$ chromosome that never recombines with the $\mathrm{X}$ experiences reduced selection, which results in an accumulation of deleterious mutations and transposable elements (Charlesworth \& Charlesworth, 2000). This accumulation of transposable elements initially leads to an increase of the size of the $\mathrm{Y}$ chromosome, which becomes larger than the $\mathrm{X}$ (Ming et al., 2011). When $Y$ degeneration progresses, genetic material can be lost without fitness costs and the $\mathrm{Y}$ may shrink (Ming et al., 2011). Therefore, after sufficient time of divergence, we may observe chromosome heteromorphy - a Y chromosome larger or smaller than the $\mathrm{X}$ chromosome, depending on the progress of degeneration (Ming et al., 2011). Classically, heteromorphy was determined using light microscopy, which is rather imprecise and size differences of $c$. $10 \%$ could be considered

*These authors contributed equally to this work homomorphic (see Divashuk et al., 2014). Although heteromorphy often corresponds to the later stages of sex chromosome evolution, it is nevertheless possible that sex chromosomes are homomorphic despite a large nonrecombining region and strong degeneration of the Y chromosome (e.g. Prentout et al., 2020). Moreover, some systems do not a evolve large nonrecombining region and stay homomorphic (Renner \& Müller, 2021).

In plants, dioecy often is of recent origin (Renner, 2014; Käfer et al., 2017), thus limiting the age of the sex chromosomes. Indeed, several rather recently evolved ( $<10 \mathrm{Myr}$ ago (Ma) homomorphic sex chromosome systems with small nonrecombining regions have been described, as in Carica papaya and Asparagus officinalis (Wu \& Moore, 2015; Harkess et al., 2017). Heteromorphic sex chromosome systems also are found, with the $\mathrm{Y}$ being larger than the $\mathrm{X}$, but recombination suppression also happened relatively recently $(<20 \mathrm{Ma})$, as in Silene latifolia and Coccinia grandis (Sousa et al., 2013; Krasovec et al., 2018; Fruchard et al., 2020).

A few cases in which dioecy evolved longer ago also exist (Käfer et al., 2017), but no strongly degenerated sex chromosomes have been described so far (Renner \& Muller, 2021). Pucholt et al, (2017) described very young sex chromosomes in Salix viminalis despite ancestral dioecy for the sister genera Salix 
and Populus. Thus, either the sex chromosomes evolved independently in different species, or there have been frequent turnovers. In the fully dioecious palm tree genus Phoenix, a sex-linked region evolved before the speciation of the 14 known species (Cherif et al., 2016; Torres et al., 2018). These sex chromosomes might be old, but do not appear to be strongly differentiated. A similar situation has been reported in the grapevine (Vitis) genus (Badouin et al., 2020; Massonnet et al., 2020), possibly because sex chromosome evolution is slowed down in such perennials with long generation time (Muyle et al., 2017).

Thus, although homologous sex chromosomes are sometimes shared between species belonging to the same genus (e.g. Silene sect. Melandrium, Phoenix) (Cherif et al., 2016; Bačovský et al., 2020), homologous sex chromosomes between different genera have never been described in plants so far. This situation is in stark contrast to the situation in animals, for which several systems are $>100 \mathrm{Myr}$ old and are shared by whole classes, for example, birds and mammals (Ohno, 1969; Fridolfsson et al., 1998; Cortez et al., 2014). Undoubtedly sex chromosomes have been less intensively studied in plants, yet there seem to be fundamental differences in the evolution of sex chromosomes in plants and animals (e.g. lack of strong sexual dimorphism in plants, discussed in Renner \& Müller, 2021). However, the extent of the differences needs to be clarified and more plant sex chromosomes need to be studied.

Dioecy very likely evolved before the genera Cannabis and Humulus split, and might even be ancestral in the Cannabaceae family (Yang et al., 2013; Zhang et al., 2019). Cannabis sativa (marijuana and hemp) is a dioecious species with nearly homomorphic XY chromosomes (with homomorphy defined as above). These sex chromosomes have a large nonrecombining region and are estimated to have started diverging between 12 and $28 \mathrm{Ma}$ (Peil et al., 2003; Divashuk et al., 2014; Prentout et al., 2020).

As for C. sativa, cytological analyses of Humulus lupulus (hop) found a XY chromosome system with a large nonrecombining region, but the $\mathrm{Y}$ chromosome is smaller than the $\mathrm{X}$ (Shephard et al., 2000; Karlov et al., 2003; Divashuk et al., 2011). The H. lupulus and C. sativa lineages split between 21 and $25 \mathrm{Ma}$ (Divashuk et al., 2014; Jin et al., 2020), which is more recently than our higher bound estimate of the age of the C. sativa sex chromosomes (28 Ma; Prentout et al., 2020). It thus is possible that the sex chromosomes of $C$. sativa and $H$. lupulus evolved from the same pair that already stopped recombining in their common ancestor, a question we address here.

As in many cultivated dioecious species, only female hop plants are harvested. Hop is used in beer brewing for its bitterness, and its production is increasing worldwide (Neve, 1991; King \& Pavlovic, 2017), mostly because of the craft beer revolution (Barth-Haas, 2019; Mackinnon \& Pavlovič, 2019). The molecule responsible for hop flower bitterness, lupulin, is concentrated in female ripe inflorescences, called cones (Okada \& Ito, 2001). In pollinated cones, the presence of seed reduces their brewing quality; because $H$. lupulus is wind pollinated, a single male plant in the hop field or its vicinity can cause broad-scale damage to the crop (Thomas \& Neve, 1976).
Usually, hop is not grown from seeds, so female-only cultures are easy to obtain, and there is no need for large-scale early sexing as in C. sativa (cf. Prentout et al., 2020). However, for varietal improvement where controlled crosses are needed, knowing the sex early might be beneficial. In $H$. lupulus, sexing is reliable 1-2 years after sowing (Patzak et al., 2002; Conway \& Snyder, 2008). A few markers have been developed, but the use of Y-specific coding sequences may increase marker quality (Patzak et al., 2002; Cerenak et al., 2019).

Here we sequenced the transcriptome of $14 \mathrm{H}$. lupulus individuals. These individuals came from a cross, from which we sequenced the parents and six offspring of each sex. We used the probabilistic approach SEX-DETECTOR, which is based on allele segregation analysis within a cross, to identify sex-linked sequences (Muyle et al., 2016). From theses analyses on $H$. lupulus and our previous results on C. sativa (Prentout et al., 2020) we describe for the first time well-differentiated sex chromosomes shared by two different genera in plants.

\section{Materials and Methods}

\section{Biological material and RNA sequencing}

As indicated in Fig. 1a, we conducted a controlled cross for sequencing. The Humulus lupulus parents, cultivar 'Wye Target' (WT; female) and the Slovenian male breeding line 2/1 (2/1), as well as six female and six male $F_{1}$ siblings (Jakše et al., 2013) were collected in July 2019 in the experimental garden of Slovenian Institute of Hop Research and Brewing, Žalec.

All offspring were phenotypically confirmed to carry either male or female reproductive organs and showed no anomalies in microsatellite genotyping data (Jakše et al., 2013). Young leaves from the laterally developing shoots were picked, wrapped in aluminium foil and flash-frozen in situ in liquid $\mathrm{N}_{2}$. Later they were pulverized and stored at $-80^{\circ} \mathrm{C}$ until RNA isolation.

Total RNA was isolated from $100 \mathrm{mg}$ frozen tissue pulverized in liquid $\mathrm{N}_{2}$ according to the protocol of Monarch Total RNA Miniprep Kit, including removal of DNA from the column with DNase I (New England Biolabs). Total RNA was quantified with QвIT 3.0, and quality was verified with the Agilent RNA Nano 6000 Kit to confirm appropriate sample RIN numbers. The total RNA samples were sent to Novogen for mRNA sequencing using Illumina's 100-bp paired end service. The data were submitted to the SRA database of the NCBI (BioSample accession SAMN17526021).

\section{Mapping, genotyping and SEX-DETECTOR}

The bioinformatic pipeline is described schematically in Fig. 1b. First, the RNA-seq data were mapped to two different references: (1) the transcriptome $H$. lupulus (obtained from the annotated genome; Padgitt-Cobb et al., 2019) and (2) the transcriptome assembly of Cannabis sativa that we also used for our previous $C$. sativa sex chromosome analysis (Supporting Information, Methods S1; Van Bakel et al., 2011; Prentout et al., 2020). For the mapping, we ran Gsnap (v.2019-09-12; Wu and Nacu, 2010; Wu et al., 
(a)

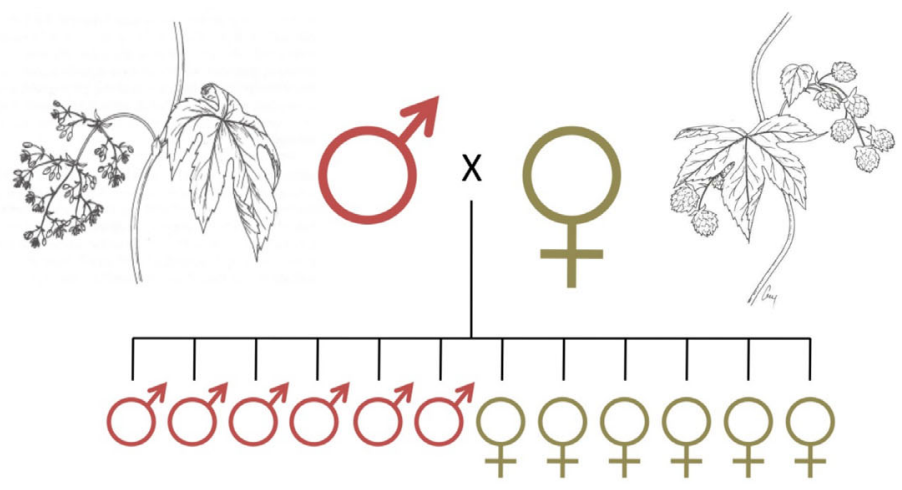

(b)

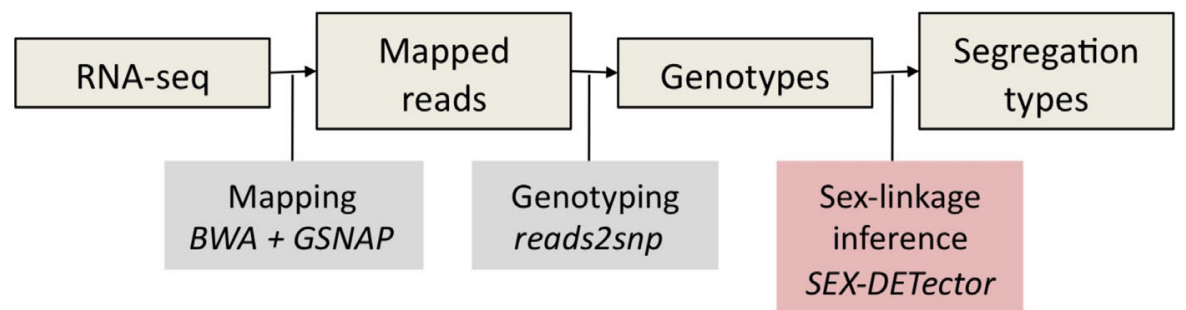

(c)

Fig. 1 Schematic representation of the workflow used to detect sex-linkage. (a) Experimental design: six females and six males were obtained by a controlled cross, and all individuals (14) were sequenced. (b) Bioinformatic pipeline for the treatment of RNA-seq data. (c) Illustration of the underlying principles of the SEX-DETECTOR segregation analysis.

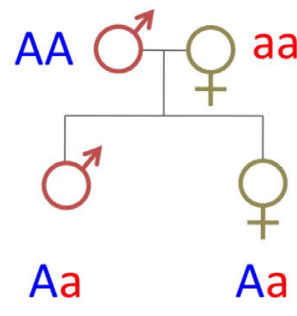

Autosomal

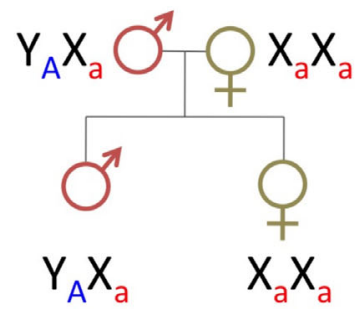

$\mathrm{X} / \mathrm{Y}$ gene pair

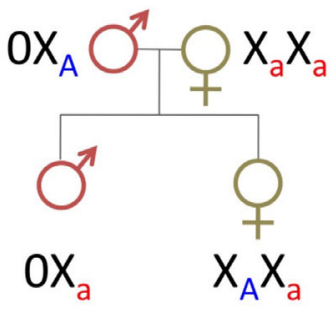

X-hemizygous
2016), an aligner that enables single nucleotide polymorphism (SNP)-tolerant mapping, with $10 \%$ mismatches allowed. This approach, already used for C. sativa analysis, was iterated several times by adding Y-specific SNPs to the references (and H. lupulusspecific SNPs while mapping on $C$. sativa reference; see Prentout et al., 2020), which increased the number of mapped reads.

Then, Samtools (v.1.4; Li et al., 2009) was used to remove unmapped reads and sort mapping output files for the genotyping. We genotyped individuals with READS2sNP (v.2.0.64; Gayral et al., 2013), as recommended for SEX-DETector (Muyle et al., 2016) - by accounting for allelic expression biases, without filtering for paralogous SNPs, and only conserving SNPs supported by at least three reads for subsequent analysis.

We ran the XY model of SEX-DETECTOR on the genotyping data, using the SEM algorithm and a threshold for an assignment of 0.8. SEX-DETECTOR computes a posterior probability of being autosomal $\left(\mathrm{P}_{\mathrm{A}}\right), \mathrm{XY}\left(\mathrm{P}_{\mathrm{XY}}\right)$ and $\mathrm{X}$-hemizygous $\left(\mathrm{P}_{\mathrm{X} \text {-hemi }}\right)$ for each SNP and for each gene (Fig. 1c). Thus, a gene with a $P_{A}$ of $\geq 0.8$ and at least one autosomal SNP without genotyping error is classified as 'autosomal'; a gene with $\mathrm{P}_{\mathrm{XY}}+\mathrm{P}_{\mathrm{X} \text {-hemi }}$ of $\geq 0.8$ and at least one sex-linked SNP without genotyping error is classified as 'sex-linked'; otherwise, the gene is classified as 'lack-ofinformation'. Among the sex-linked genes, we classified a gene as X-hemizygous if it fulfilled one of these two criteria: (1) the gene carried only X-hemizygous SNPs and at least one SNP without genotyping error, (2) the $\mathrm{Y}$ expression of the gene is detected only from positions with genotyping errors. A parameter that is important to optimize with SEX-DETECTOR is the Y-specific genotyping error rate ( $p$; see Muyle et al., 2016). However, the quantity of Ylinked reads that map on a female reference diminishes with $\mathrm{X}-\mathrm{Y}$ divergence; therefore, old and highly divergent sex chromosomes are more susceptible to mapping errors and thus genotyping errors. $p$ is expected to be close to the whole transcriptome genotyping error rate $(\epsilon)$, but could be higher as a consequence of weak expression (resulting in less reads) of the Y-linked copies or to mapping on a divergent $\mathrm{X}$ reference. To reduce the gap between these two error rates, we ran four iterations with GSNAP, using at each time the SNPs file generated by SEX-DETector. This SNPs file 
contains $H$. lupulus-specific polymorphisms, initially absent from the $C$. sativa reference transcriptome, and increased the quantity of mapped reads by adding these SNPs to the reference and, thus, fitting it with the $H$. lupulus RNA-seq.

As detailed in Methods $\mathrm{S} 1$, we retained the mapping on $C$. sativa transcriptome assembly for downstream analysis. Indeed, the mapping of Y-linked reads and SEX-DETECTOR results obtained with a mapping on C. sativa reference transcriptome were more robust than those obtained with a mapping on H. lupulus reference transcriptome (Methods S1).

\section{Sex-linked gene positions on C. sativa genome}

As the $H$. lupulus RNA-seq data were mapped on the $C$. sativa transcriptome, we determined the position of the transcript sequences from the $C$. sativa transcriptome assembly (van Bakel et al., 2011) on a chromosome-level assembly of the C. sativa genome (Grassa et al., 2018) with BLAST (v.2.2.30+; Altschul et al., 1990). We selected the best hit with an e-value lower than $10^{-4}$ to determine the position of the transcript on the genome. Then, we split each chromosome in windows of $2 \mathrm{Mb}$ and computed the density of sex-linked genes and nonsex-linked genes per window using BeDTOols (v.2.26.0; Quinlan \& Hall, 2010). Proportions of sex-linked genes were computed by dividing the number of sex-linked genes by the total number of genes (sexlinked, autosomal and undetermined) in the same window. For C. sativa, densities were already available from our previous analysis (Prentout et al., 2020).

\section{Molecular clock and age of sex chromosomes}

We used the translated reference transcripts (van Bakel et al., 2011) to determine the $X$ and $Y$ Open Reading Frame (ORF) of nucleotide reference transcripts. For each XY gene pair, the $d S$ values were estimated with codeml (PAmL v.4.9; Yang, 2007) in pairwise mode. Then, we used two molecular clocks, derived from Arabidopsis species, to estimate the age of H. lupulus sex chromosomes (Koch et al., 2000; Ossowski et al., 2010). In the wild, $H$. lupulus flowers in the second or third year of development (Patzak et al., 2002; Polley et al., 1997), therefore, we took a generation time (GT) of 2 years, and used the molecular clocks as follows: $(d S) /$ rate $=d S /\left(1.5 \times 10^{-8}\right)$ using the molecular clock from Koch et al., (2000); $(\mathrm{GT} \times d S) /(2 \times \mu)=d S /\left(7 \times 10^{-9}\right)$ using the clock from Ossowski et al., (2010). Three different estimates of $d S$ were used: the maximum $d S$ value, the mean of the $5 \%$ highest $d S$ values, and the mean of the $10 \%$ highest $d S$ values.

\section{$X$ and $Y$ allele-specific expression analysis}

In addition to identifying $X$ and $Y$ alleles, SEX-DETECTOR estimates their expression based on the number of reads (Muyle et al., 2016). These estimates rely on counting reads spanning XY SNPs only and were normalized using the total read number in a library for each individual. We further normalized them by the median autosomal expression for each individual. The C. sativa results presented here were generated in our previous analysis on C. sativa sex chromosomes (Prentout et al., 2020).

\section{Correction of $\mathrm{Y}$ read mapping bias}

The use of a female reference for the mapping of the reads might create mapping biases, resulting in the absence of $\mathrm{Y}$ reads in the most diverging parts of the genes. This issue may reduce the divergence detected and change the phylogenetic signal (Dixon et al., 2019). If, within a same gene, regions that lack Y reads coexist with regions where the Y reads correctly mapped, we expect to see a signature similar to gene conversion (i.e. regionwise variation in divergence). Therefore, we ran GENECONV (v.1.81a; Sawyer, 1999) in pairwise and group mode with the multiple alignments used for the phylogeny (on 85 gene alignments before Gblock filtering, see below) in order to identify and remove regions with reduced divergence. We defined two groups, one for $\mathrm{X}$ and $\mathrm{Y}$ sequences in $H$. lupulus, and the other one for $\mathrm{X}$ and $\mathrm{Y}$ sequences in $C$. sativa. Then, we conserved only inner fragments and split the gene conversion regions from regions without gene conversion to obtain two subsets per gene. Thus, we obtained a subset of sequences corrected for the mapping bias, in addition to the set of genes not filtered with GENECONV.

\section{Phylogenetic analysis}

We reconstructed gene families for genes identified as sex-linked in both C. sativa and H. lupulus. Then, we used BLASTP, filtering for the best hit (with an e-value threshold fixed at $10^{-4}$ ), to find homologous sequences between $C$. sativa reference transcripts (the query sequence in BLASTP) (van Bakel et al., 2011) and four outgroup transcriptomes (the subject sequence in BLASTP): Trema orientalis (Cannabaceae; van Velzen et al., 2018), Morus notabilis (Moraceae; He et al., 2013), Fragaria vesca ssp. vesca (Rosaceae; Shulaev et al., 2011) and Rosa chinensis (Rosaceae; Raymond et al., 2018). Gene families for which at least two outgroup sequences have been identified were kept, other gene families were discarded from subsequent analysis. Then, we added X and Y sequences reconstructed by SEX-DETECTOR to each gene family. To distinguish potential paralogous sequences or variants from alternative splicing, a blast of all sequences vs all sequences was realized. If two sequences from two distinct gene families matched with each other (with an e-value threshold fixed at $10^{-4}$ ), then both families were removed from the dataset. Finally, we retrieved the corresponding nucleotide sequence of each protein, which constituted the dataset used for the phylogenetic analysis.

Using MACSE (v.2.03; Ranwez et al., 2011), and before alignment, nonhomologous segments of $\geq 60$ nucleotides within or 30 nucleotides at the extremity of a nucleotide sequence were trimmed if they displayed $<30 \%$ of similarity with other sequences from the gene family. This step allowed the removal of misidentified outgroup sequences. Then, gene families with no remaining outgroup sequences were discarded. Finally, remaining families were aligned with MACSE, allowing sequences to be removed and realigned, one sequence at a time and over multiple iterations, to improve local alignment. 
Nucleotide alignments were cleaned at the codon level using GBLOCKS (with default parameters) to conserve only codons shared by all sequences (v.0.91b; Castresana, 2000). For maximumlikelihood (ML) phylogenetic tree reconstruction, we used ModelFinder in IQ-TReE (v.1.639; Nguyen et al., 2015; Kalyaanamoorthy et al., 2017) to select the best-fit substitution model for each alignment. Those models were then used in RAxML-NG (v.1.0.0; Kozlov et al., 2019) to reconstruct gene family trees. The number of bootstrap replicates was estimated using AUTOMRE (Pattengale et al., 2010) criterion (maximum 2000 bootstraps). The ML phylogenetic tree reconstruction was run on two datasets, one without removing potential mapping biases, and one with the potential mapping bias removed, as described above.

Bayesian phylogenies were built using PhyLOBAYES (v.3.4; Lartillot et al., 2009) with the site-specific profiles CAT and the CAT-GTR models with a gamma distribution to handle across site rate variations. Two chains were run in parallel for a minimum of 500 cycles. The convergence between the two chains was checked every 100 cycles (with a burn-in equal to one fifth of the total length of the chains). Chains were stopped once all the discrepancies were $\leq 0.1$ and all effective sizes were $>50$ and used to build a majority rule consensus tree.

\section{Statistics and linear chromosome representations}

The statistical analyses were conducted with $\mathrm{R}$ (v.3.4.4; R Core Team, 2018). We report exact $P$-values when they are $>10^{-5}$.
The representation of phylogenetic topologies, $d S$ values on the first chromosome and the dosage compensation graphics have been done with GgPLOT2 (Wickham, 2011). For the circular representation of the sex-linked gene density along the $C$. sativa genome we used the CIRCLIZE package in R (GU et al., 2014). The correspondence between names in Fig. 2a and names in the genome assembly is indicated in Table S1. We calculated confidence intervals for the median of a dataset of $n$ observations by resampling 5000 times $n$ values from the dataset (with replacement). The confidence intervals are then given by the quantiles of the distribution of median values obtained by resampling.

\section{Results}

\section{Identification of sex-linked genes in $\mathrm{H}$. lupulus}

As mentioned in the Materials and Methods section, we used the mapping of the $H$. lupulus RNA-seq data on the C. sativa transcriptome assembly for downstream analysis. Of the 30074 genes in the C. sativa reference transcriptome, 21268 had detectable expression in our $H$. lupulus transcriptome data. The difference of properly-paired mapped reads between males (mean $32.3 \%$ ) and females (mean 34.9\%) was slightly significant (Wilcoxon's test two-sided $P$-value $=0.038$; see Table S2), which may be explained by a reduced mapping efficiency of Y-linked reads on the female reference. (a)

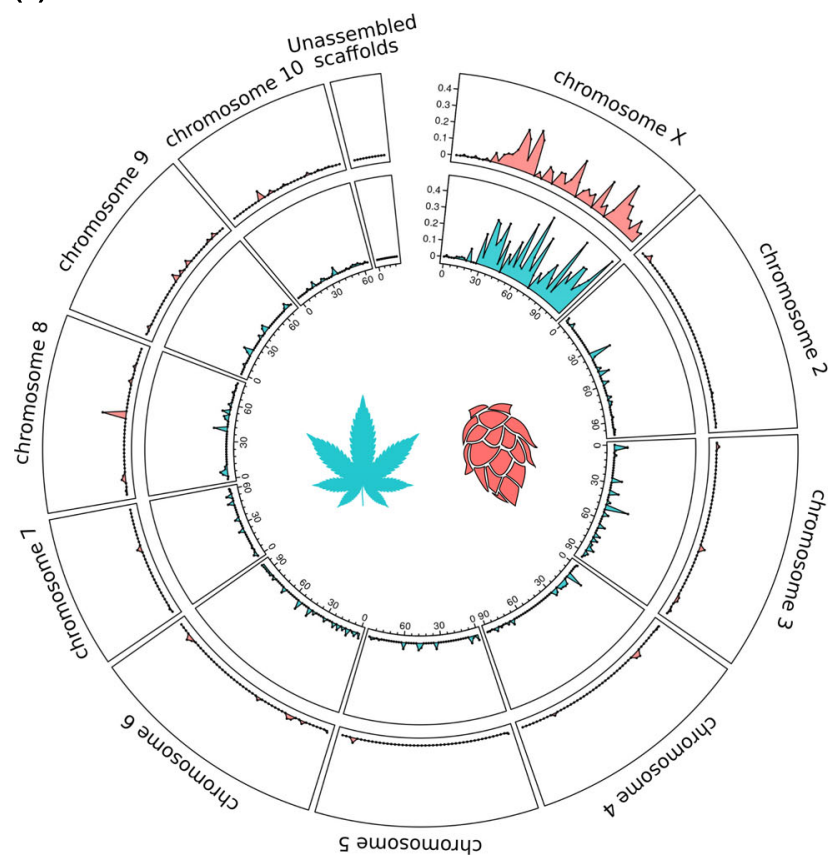

(b)

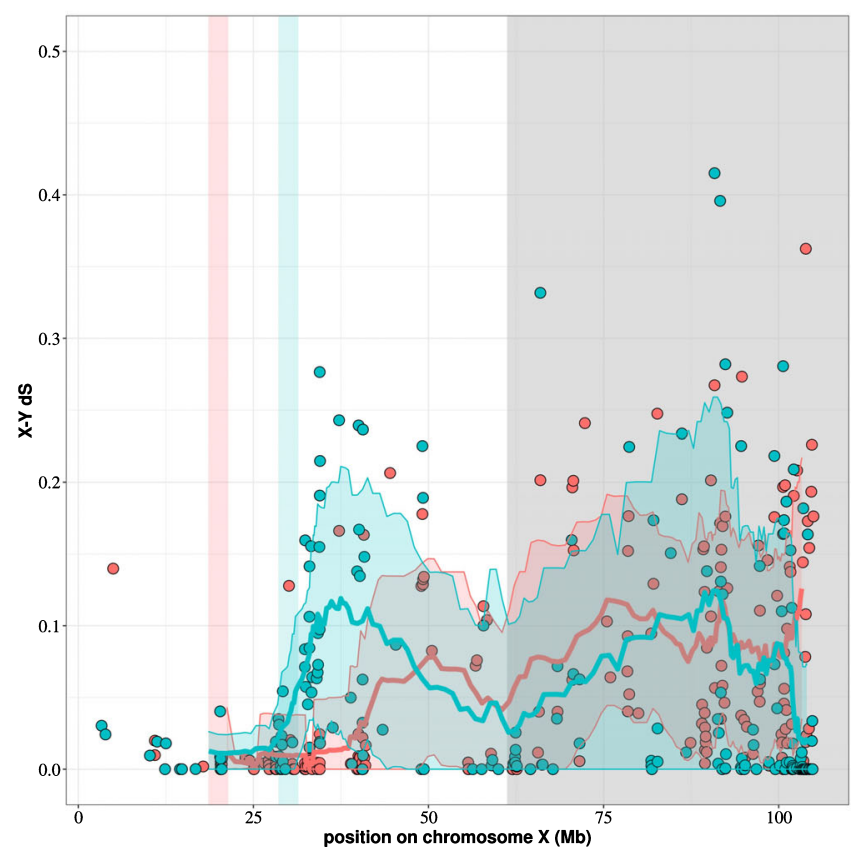

Fig. 2 (a) Humulus lupulus sex-linked genes mapped on the Cannabis sativa genome (Grassa et al., 2018). Inner graphs (in blue): C. sativa sex-linked gene density corrected by the total gene density in 2-Mb windows (from Prentout et al., 2020). Outer graphs (in red): H. lupulus sex-linked gene density corrected by the total gene density in 2-Mb windows. (b) Synonymous divergence ( $d S$ ) between $X$ and $Y$ copies of $H$. lupulus sex-linked genes (red) and those of $C$. sativa (blue) along the $X$ chromosome of $C$. sativa. The curves represent the average $d S$ with sliding windows (windows of 20 points), for $H$. lupulus (red) and C. sativa (blue). Confidence intervals (average \pm SD) are indicated around the $H$. lupulus curve (red area) and the $C$. sativa curve (blue area). The vertical red bar represents the putative Pseudo-Autosomal Boundary (PAB) in $H$. lupulus, the vertical blue bar represents the putative PAB in $C$. sativa, the grey area represents the region that stopped recombining in a common ancestor. 
The sex-linked sequences from $H$. lupulus transcriptome data were identified with SEX-DETector (Muyle et al., 2016). It is important that genotyping error rate parameters $\epsilon$ and $p$ have similar values ( $\epsilon$, whole transcriptome; $p, \mathrm{Y}$ chromosome) to obtain reliable SEX-DETeсTOR outputs. At the fourth iteration of GsNaP mapping on C. sativa reference transcriptome $\epsilon$ and $p$ stabilized at 0.06 and 0.20 , respectively (Table S3). Upon closer inspection, one H. lupulus male (\#3) appeared to have many genotyping errors, as for some $\mathrm{XY}$ genes, this male was genotyped as both heterozygous (XY) and homozygous (XX), which increased the error rate $p$. The identification of Y SNPs with this individual RNA-seq data discarded the hypothesis of a mislabelled female or a XX individual that developed male flowers. A particularly strong $Y$ reads mapping bias in this male may explain these observations. After removal of this male, the error rate $p$ dropped to 0.10 (Table S3). A total of 265 sex-linked genes were identified in $H$. lupulus, which represents $7.8 \%$ of all assigned genes (autosomal genes + sex-linked genes; Table 1). The SEXDETeCTOR assignment output is provided in Table S4.

\section{$H$. lupulus and C. sativa sex chromosomes are homologous}

Among $265 H$. lupulus $\mathrm{XY}$ genes from the $C$. sativa transcriptome assembly (van Bakel et al., 2011), 254 genes are present on the $C$. sativa chromosome-level genome assembly (Grassa et al., 2018). As shown in Fig. 2a, 192 of these genes (75.6\%) map on C. sativa chromosome number 1 , which is the chromosome we previously identified as the $\mathrm{X}$ chromosome in C. sativa (Prentout et al., 2020). Of the 265 sex-linked genes in $H$. lupulus, 112 also were detected as sex-linked in C. sativa, whereas 64 were detected as autosomal and 89 had unassigned segregation type (Prentout et al., 2020).

The synonymous divergence $(d S)$ between $\mathrm{X}$ and $\mathrm{Y}$ copies of the sex-linked genes of $H$. lupulus was distributed in a similar way along the $C$. sativa sex chromosome as the values for this latter species, as shown in Fig. 2b. Although the sampling variation of these $d S$ values is large, as expected (cf. Takahata \& Nei, 1985), it can be observed that the larger values occurred in the region beyond $65 \mathrm{Mb}$.

Table 1 Summary of the SEX-DETECTOR results.

\begin{tabular}{lr}
\hline & Number \\
\hline All genes* & 30074 \\
Expressed genes & 21268 \\
Genes with SNPs used by SEX-DETECTOR & 4472 \\
Genes with undetermined segregation type class 1** & 462 \\
Genes with undetermined segregation type class 2*** & 354 \\
Autosomal genes & 3391 \\
Sex-linked genes & 265 \\
XY genes & 265 \\
X-hemizygous genes & 0 \\
\hline
\end{tabular}

*Transcripts from gene annotation of the $C$. sativa reference genome (van Bakel et al., 2011).

$* *$ Posterior probabilities $<0.8$

***Posterior probabilities $>0.8$ but absence of single nucleotide polymorphisms (SNPs) without error.

\section{$\mathrm{X}-\mathrm{Y}$ recombination likely stopped before the Cannabis and Humulus genera split}

We reconstructed phylogenetic trees of genes detected as sexlinked in both species, including outgroup sequences from the order Rosales. For 27 of the 112 sex-linked genes present in both species, we could not identify any homologous sequences in the outgroup species and those genes were excluded from further analysis. For the remaining 85, we determined the topology of the gametologous sequences in the Cannabaceae, considering a node to be well-resolved when the bootstrap support exceeded $95 \%$, or Bayesian support exceeded 0.95 .

The three different methods for phylogenetic reconstruction provided consistent phylogenies (Table 2). More precisely, we observed three major topologies, as shown in Fig. 3: X copies of both species form a clade separated from a clade of $\mathrm{Y}$ sequences (topology I; Fig. 3a), the $\mathrm{X}$ and $\mathrm{Y}$ sequences of each species group together (topology II; Fig. 3b), or a paraphyletic placement of the $\mathrm{X}$ and $\mathrm{Y}$ sequences of $H$. lupulus, relative to $C$. sativa sequences (topology III; Fig. 3c). As shown in Table 2, we found that most genes had topology II, corresponding to recombination suppression after the split of the genera. A few genes, however, had topology I, which corresponds to genes for which recombination already was suppressed in a common ancestor of both species. As shown in Fig. 3(d), topologies I and III occurred mainly beyond $80 \mathrm{Mb}$, whereas topology II occurred all over the chromosome. Topology I is associated with higher synonymous divergence.

We identified 42 genes, of the 85 genes used for the phylogeny, with at least one fragment in at least one species that displayed reduced divergence (with a $P$-value $<0.05$ in GENECONV output). Because this reduction of divergence may be caused by a mapping bias of $Y$ reads, we ran the ML phylogenetic reconstruction method on regions with and without mapping bias (example in Fig. S1). As shown in Table 2 and Fig. 3(e), after mapping bias filter with GENECONV, the proportion of genes displaying topology $\mathrm{I}$, indicating recombination suppression in a common ancestor, increased, whereas fewer genes with topology II were found, mainly in a restricted region corresponding to the region where recombination stopped independently in both species.

This leads us to define three regions on the $\mathrm{X}$ chromosomes of $C$. sativa and $H$. lupulus (with the $C$. sativa $\mathrm{X}$ chromosome as a reference). A region from $c .65 \mathrm{Mb}$ to the end of the $\mathrm{X}$ chromosome that already stopped recombining in a common ancestor; from c. 20-30 $\mathrm{Mb}$ to $c .65 \mathrm{Mb}$, a part of the nonrecombining region that evolved independently in the two species; and from the beginning of the chromosome to $c .20-30 \mathrm{Mb}$, the pseudoautosomal region (PAR), where few sex-linked genes are found.

\section{Age of $H$. lupulus sex chromosomes}

In order to estimate the age of the sex chromosomes, we used the maximum synonymous divergence between $\mathrm{X}$ and $\mathrm{Y}$ sequences and two molecular clocks, which were both derived from Arabidopsis. Because the sampling variance in $d S$ values can be large, we used three ways to calculate the maximum $d S$ value: the single highest $d S$ 
Table 2 Results of the phylogenetic reconstruction of sex-linked genes.

\begin{tabular}{|c|c|c|c|c|c|c|}
\hline & $\begin{array}{l}\text { Topology I } \\
\left(\left(X_{c}, X_{h}\right),\left(Y_{c}, Y_{h}\right)\right)\end{array}$ & $\begin{array}{l}\text { Topology II } \\
\left(\left(X_{c}, Y_{c}\right),\left(X_{h}, Y_{h}\right)\right)\end{array}$ & $\begin{array}{l}\text { Topology III } \\
\left(Y_{h r}\left(X_{h},\left(X_{c}, Y_{c}\right)\right)\right)\end{array}$ & Other & Unresolved & Total \\
\hline Maximum-likelihood (ML) & 7 & 44 & 7 & 1 & 26 & 85 \\
\hline GTR (Bayesian) & 4 & 45 & 4 & 8 & 24 & 85 \\
\hline CAT-GTR (Bayesian) & 4 & 44 & 7 & 7 & 23 & 85 \\
\hline ML after GENECONV filtering & 11 & 27 & 11 & 4 & 32 & 85 \\
\hline
\end{tabular}

Phylogenetic trees with a bootstrap value equal or greater than $95 \%$ (and posterior probabilities higher than 0.95 for Bayesian reconstructions) at the node separating Cannabis sativa and Humulus lupulus, or $\mathrm{Y}$ and $\mathrm{X}$ sequences, are presented in the first four columns. Phylogenetic trees without such support are classified as 'unresolved'.

(d)

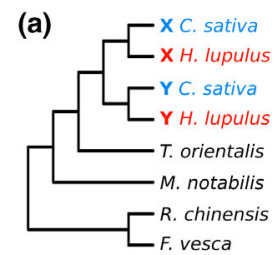

(b) $\square_{Y \text { C. sativa }}^{x \text { cativa }}$

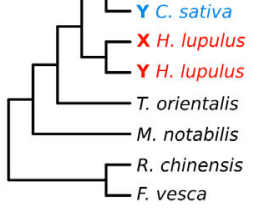

(c) $--^{\times C}$. sativa

(c) $\left[\begin{array}{l}x \\ \mathbf{x} \text { C. sativa }\end{array}\right.$

Y Y. lupulus

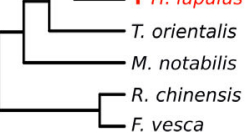

- R. vesca
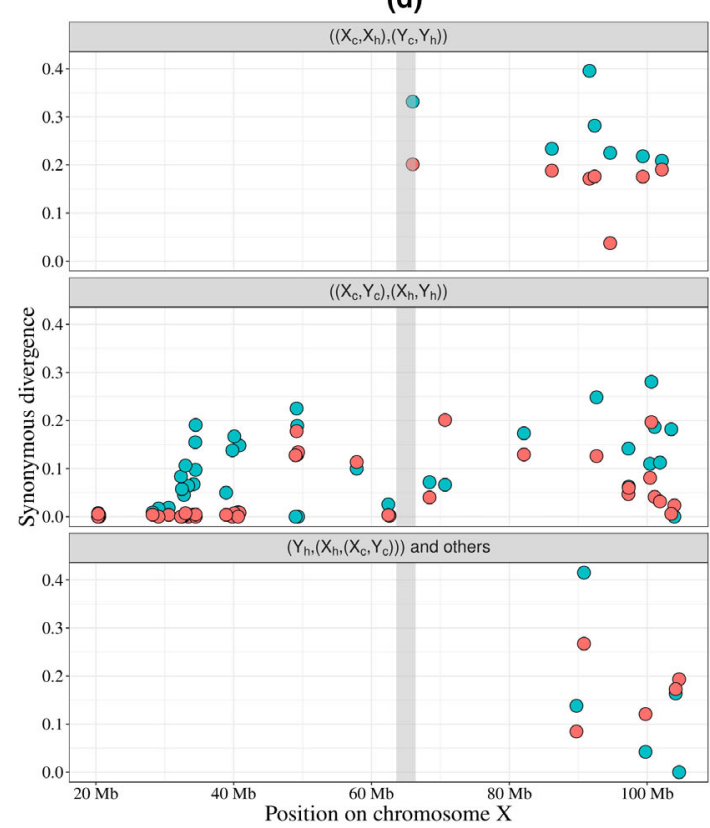

(e)

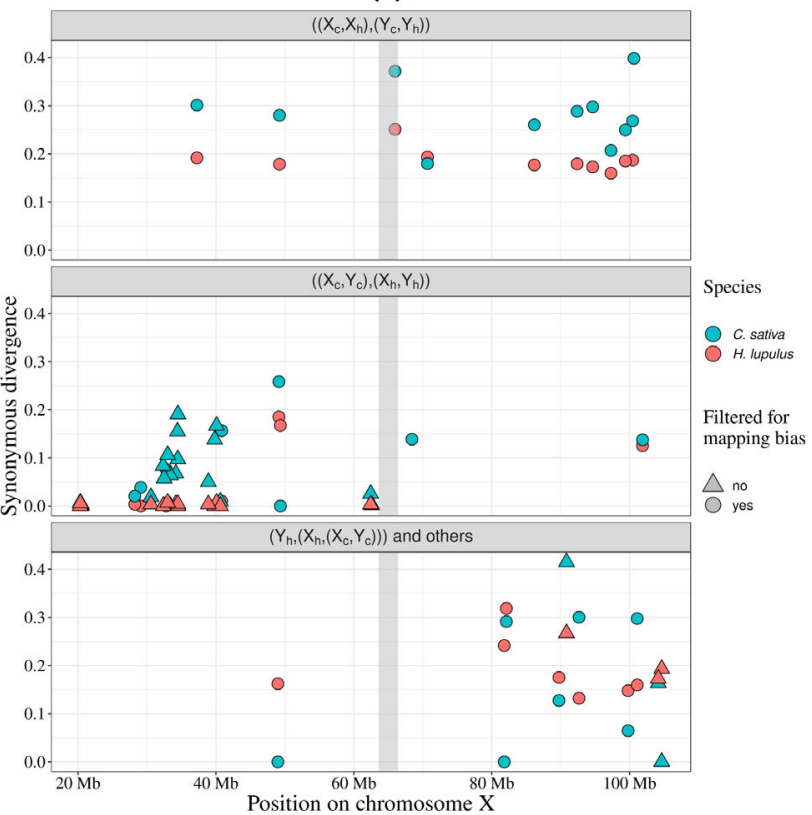

Fig. 3 Distribution of the three topologies of the sex-linked genes on the $X$ chromosome: (a) Topology I, XX-YY - arrest of recombination before the split of the two genera, (b) Topology II, XY-XY - arrest of recombination after the split of the two genera, (c) Topology III, Y-X-XY - Humulus lupulus X chromosome is closer to Cannabis sativa sequences than its $Y$ counterpart. (d) Distribution of the topologies along the $C$. sativa $X$ chromosome ('other' topology is included in the $Y-X-X Y$ topology panel), using the full gene sequences. For each gene, dots represent the $d S$ values in $C$. sativa (blue) and $H$. lupulus (red). (e) Distribution of the topologies after filtering out possible mapping biases through geneconv. Triangles indicate that at least one segment was removed, dots indicate sequences for which no mapping bias was detected. The vertical grey bar (d,e) represents the putative boundary between the region that stopped recombining in a common ancestor and the region that stopped recombining independently in the two species.

value; the average of the $5 \%$ highest values; and the average of the $10 \%$ highest values. Furthermore, we calculated these on the raw alignments as well as the alignments with possible mapping biases removed. The different estimates are given in Table 3, and yielded values between 14.5 and $51.4 \mathrm{Ma}$. The minimum synonymous divergence between $C$. sativa and outgroup species Morus notabilis and Rosa chinensis was c. 0.45 and c. 0.65, respectively (Figs S2, S3), higher than the maximum synonymous divergence between sexlinked gene copies, indicating that the sex chromosomes probably evolved in the Cannabaceae family.

\section{Y gene expression}

The $\mathrm{Y}$ over $\mathrm{X}$ expression ratio is a standard proxy for the degeneration of the $\mathrm{Y}$ chromosome. A Y/X expression ratio close to 1 means no degeneration, a $\mathrm{Y} / \mathrm{X}$ expression ratio close to 0.5 or below means strong degeneration. In H. lupulus, the median $\mathrm{Y} / \mathrm{X}$ expression ratio was 0.637 (Fig. S4), which is significantly different from $1\left(99^{\text {th }}\right.$ percentile of median distribution with 5000 samples in initial distribution $=0.673)$. The median was not different when considering all sex-linked genes (0.637) or only the sex-linked genes mapping on C. sativa X chromosome $(0.639, P=0.70$, onesided Wilcoxon rank sum test).

In both species, the reduced $\mathrm{Y}$ expression is correlated to the position on the $\mathrm{X}$ chromosome (linear regression: adjusted $R^{2}=$ $0.134, P<10^{-5}$ for H. lupulus, and adjusted $R^{2}=0.278$, $P<10^{-5}$ for C. sativa). As shown in Fig. 4, the Y/X expression ratio decreased while moving away from the PAR in $H$. lupulus, and this also was confirmed in C. sativa. 
Table 3 Age estimates with two molecular clocks and different maximum synonymous divergence values (dS values).

\begin{tabular}{|c|c|c|c|c|c|c|}
\hline & \multicolumn{3}{|c|}{ No filtering } & \multicolumn{3}{|c|}{ Mapping bias filtering } \\
\hline & $d S$ & Age $(\text { Myr old })^{1}$ & Age $(\text { Myr old })^{2}$ & $d S$ & Age $(\text { Myr old })^{1}$ & Age $(\text { Myr old })^{2}$ \\
\hline Highest $d S$ & 0.362 & 51.4 & 24.0 & 0.362 & 51.4 & 24.0 \\
\hline Mean highest $5 \%$ & 0.249 & 35.6 & 16.6 & 0.274 & 39.1 & 18.3 \\
\hline Mean highest 10\% & 0.217 & 31.0 & 14.5 & 0.214 & 34.4 & 16.1 \\
\hline
\end{tabular}

For each $d S$ value, two ages were obtained using the molecular clocks of ${ }^{1}$ Ossowski et al. (2010) and ${ }^{2}$ Koch et al. (2000). Two alignment datasets were used, with or without filtering for possible mapping bias.

(a)

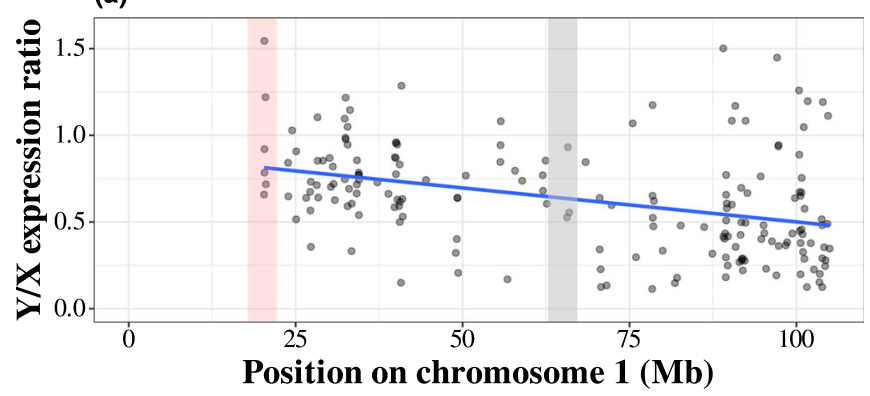

(b)

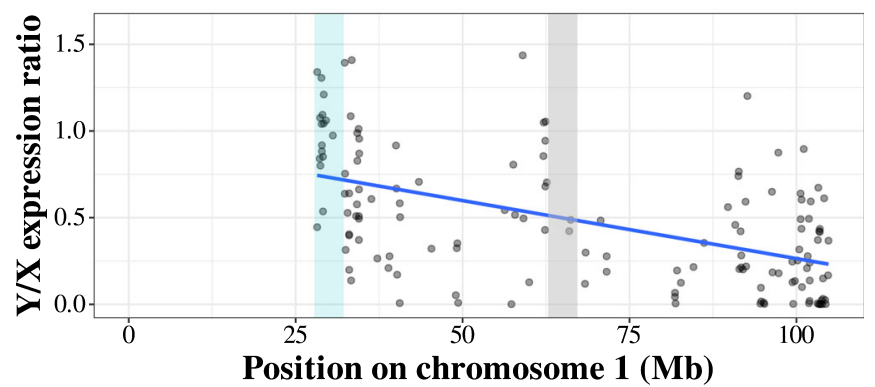

Fig. $4 \mathrm{Y} / \mathrm{X}$ expression ratio along the nonrecombining region in Humulus lupulus (a) and Cannabis sativa (b). Each dot is the $Y / X$ expression ratio for one gene in the nonrecombining region only (the linear regression result is indicated by the blue line). The vertical red bar represents the putative $P A B$ in $H$. lupulus, the vertical blue bar represents the putative $P A B$ in $C$. sativa, the vertical grey bar represents the putative boundary between the region that stopped recombining in a common ancestor and the region that stopped recombining independently in the two species.

\section{Dosage compensation}

We tested whether the expression of the $\mathrm{X}$ chromosome changed following degeneration of the $\mathrm{Y}$ chromosome, a phenomenon called dosage compensation (Muyle et al., 2017). We used the ratio of the male $\mathrm{X}$ expression over the female XX expression as a proxy for dosage compensation (Muyle et al., 2012) and Y/X expression ratio as a proxy for $\mathrm{Y}$ degeneration. Genes with strong degeneration (Y/X expression ratio close to zero) displayed an increased expression of the $\mathrm{X}$ in males (linear regression: adjusted $R^{2}=0.179, P<10^{-5}$ and adjusted $R^{2}=0.097, P<10^{-5}$ for H. lupulus and C. sativa, respectively), as shown in Fig. 5. A dosage compensation pattern was found in both in $H$. lupulus and C. sativa in agreement with previous work (Prentout et al., 2020).

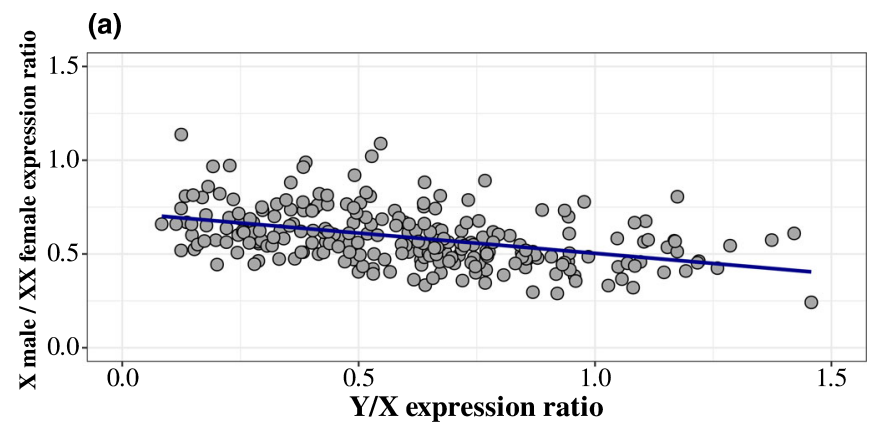

(b)

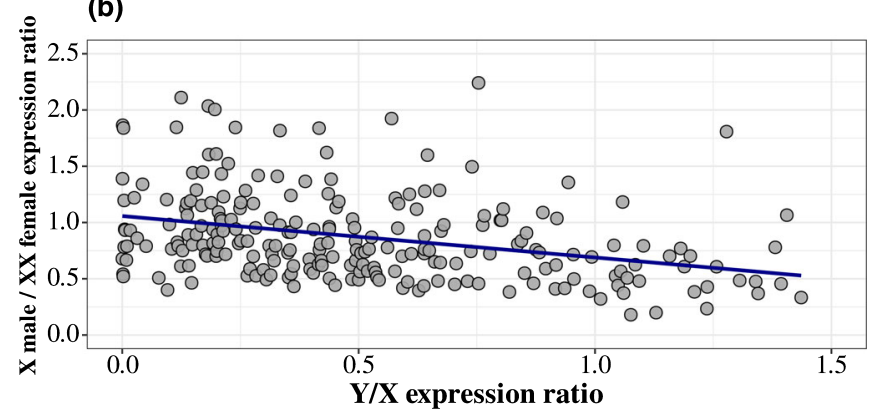

Fig. 5 The male $X$ expression over female $X X$ expression versus $Y / X$ expression ratio for Humulus lupulus (a) and Cannabis sativa (b). Each black dot represents one gene. The blue line represents a linear regression.

\section{Discussion}

We here identified the Humulus lupulus sex chromosomes, and found that they are homologous to those of Cannabis sativa (Prentout et al., 2020), and that a part of these chromosomes had already stopped recombining in a common ancestor of the two species. Performing a segregation analysis with SEX-DETECTOR (Muyle et al., 2016), we identified $265 \mathrm{XY}$ genes in H. lupulus, among which 112 also were sex-linked in C. sativa. Mapping these genes on the chromosome-level assembly of $C$. sativa (Grassa et al., 2018) suggested that the nonrecombining region is large in $H$. lupulus, as proposed before based on cytological studies (Divashuk et al., 2011).

We identified three different regions on the sex chromosome, based on the distribution of sex-linked gene phylogenetic topologies and synonymous divergence between the $\mathrm{X}$ and $\mathrm{Y}$ copies on the $C$. sativa $\mathrm{X}$ chromosome: one region that had already stopped recombining in a common ancestor of $C$. sativa and $H$. lupulus, a region that independently stopped recombining in both species, 
and the pseudo-autosomal region. Our results suggest the pseudo-autosomal boundary (PAB) in $H$. lupulus may be located around $20 \mathrm{Mb}$, whereas we estimated a PAB around $30 \mathrm{Mb}$ in $C$. sativa (Prentout et al., 2020); the nonrecombining region thus may be larger in $H$. lupulus than in $C$. sativa. With this estimation of the size of the nonrecombining region in $H$. lupulus, among the 3469 genes present on the X chromosome, 2045 genes would be located in this nonrecombining region (which represents $59.1 \%$ of all the genes on the $\mathrm{X}$ chromosome). However, a chromosome-level assembly of the $H$. lupulus genome would be needed to determine the exact position of the PAB in this species, as synteny might not be fully conserved. In addition, because we used one single cross, it is possible that we overestimated the size of the nonrecombining region as a consequence of linkage disequilibrium. Thus, genes around the PAB classified as sex-linked and for which we estimated a low $d S$ value may still be recombining. An accurate estimation of the $\mathrm{PAB}$, as has been done for example in Silene latifolia, would require much more offspring and data from several populations (Krasovec et al., 2020).

Several sex-linked genes had topologies that were not compatible with either recombination suppression in a common ancestor or in each of the species independently. Strikingly, most of these topologies placed the $H$. lupulus $Y$ sequence as an outgroup to the other sex-linked gene sequences. Whether this is the result of errors (e.g. long-branch attraction, mapping biases) remains to be investigated. Interestingly, genes with theses 'unexpected' topologies all clustered (except for one gene) in a region of $c .25 \mathrm{Mb}$. This region is located at the extremity of the $\mathrm{X}$ chromosome, which, as we suggested, stopped recombining first. It is likely to observe a high rate of unexpected phylogenetic results in the region that stopped recombining first because the $\mathrm{X}-\mathrm{Y}$ divergence should be the highest in this region, which could increase the mapping bias. Our approach to correct for the $\mathrm{Y}$ read mapping relies on GENECONV, which is known to have a high rate of false negatives (Lawson \& Zhang, 2009). This also could explain the unexpected presence of some of the XY-XY genes in the older region.

$\mathrm{X}-\mathrm{Y}$ gene conversion has been shown to affect only a few genes in animals (Katsura et al., 2012; Trombetta et al., 2014; Peneder et al., 2017). Although we do not expect gene conversion for half of the genes that are sex-linked in both species, it is worth noting that a part of fragments identified by GENECONV may correspond to real gene conversion rather than mapping biases. Here again, assemblies of $\mathrm{Y}$ and $\mathrm{X}$ chromosomes in both species are required to determine the presence of true $\mathrm{X}-\mathrm{Y}$ gene conversion.

The highest $d S$ values and the genes with a topology indicating that recombination was already suppressed in the common ancestor are located in the same region $(65 \mathrm{Mb}$ to the end of the chromosome). These results suggest the presence of at least two strata in these sex chromosomes. We estimated that the youngest stratum is 10.1-29.4 Myr old in H. lupulus, and 15.9-19.8 Myr old in C. sativa (Table S5). However, although recombination suppression clearly did not occur for all of the sex-linked genes at the same time, we cannot determine the exact number of strata in the sex chromosomes of C. sativa and H. lupulus. It also is possible that recombination was suppressed gradually, with the recombination suppression starting before the split of both genera and continuing afterwards. To clearly determine the number of strata, an identification of chromosomal inversions or significative differences in $d S$ values along the sex chromosomes is required (Nicolas et al., 2004; Lemaitre et al., 2009; Wang et al., 2012; reviewed in Wright et al., 2016). Thus, X and Y chromosome assemblies for both $H$. lupulus and $C$. sativa are needed to exactly determine the number (and location) of strata in both species. Moreover, a Y chromosome assembly will allow the identification of Y-specific genes, which is not possible with SEXDETECTOR and the data that we used.

We did not find X-hemizygous genes in H. lupulus. This is striking as $218 \mathrm{X}$-hemizygous genes (38\% of all sex-linked genes) were found in C. sativa using the same methodology (Prentout et al., 2020). A very low level of polymorphism could result in the inability of SEX-DETECTOR to identify X-hemizygous genes (Muyle et al., 2016), but in that case SEX-DETECTOR also should have problems identifying autosomal genes, which was not the case here. Nonrandom X-inactivation in females could be an explanation, as the nonrandom expression of a single $\mathrm{X}$ allele in females would impede SEX-DETECTOR to identify X-linkage and X-hemizygous genes (Muyle et al., 2016). We ran an AlleleSpecific Expression (ASE) analysis, which did not support this hypothesis (Figs S5-S7). Humulus lupulus probably is an ancient polyploid that reverted to the ancestral karyotype (Padgitt-Cobb et al., 2019). It is thus possible that the H. lupulus $\mathrm{X}$ chromosome comprises two copies of the ancestral $\mathrm{X}$ as some cytological data seem to suggest (Divashuk et al., 2011). In this case, SEXDETECTOR would manage to identify the XY gene pairs, but would fail to identify the $\mathrm{X}$-hemizygous genes as these genes would exhibit unexpected allele transmission patterns (Fig. S8).

Humulus lupulus is a rare case of XY systems in plants in which the $\mathrm{Y}$ is smaller than the $\mathrm{X}$ (cf. Ming et al., 2011). In C. sativa, both sex chromosomes have similar sizes (Divashuk et al., 2014). If the size difference is caused by deletions of parts of the $H$. lupulus $\mathrm{Y}$ chromosome, which is the hypothesized mechanism in many species (cf. Ming et al., 2011), we expect to observe that many $\mathrm{XY}$ gene pairs in $C$. sativa have missing $\mathrm{Y}$ copies in $H$. lupulus. As explained above, we did not detect any $\mathrm{X}$ hemizygous genes. Furthermore, the XY gene pairs of $H$. lupulus were distributed uniformly on the C. sativa $\mathrm{X}$ chromosome, and no region appeared to be depleted in $\mathrm{XY}$ genes, which is not what we would observe if large deletions were present on the $H$. lupulus $\mathrm{Y}$ chromosome. The sex chromosome size differences observed in $H$. lupulus probably reflect complex dynamics, different from that of old animal systems with tiny $\mathrm{Y}$ chromosome resulting from large deletions (e.g. Skaletsky et al., 2003; Ross et al., 2005). The large size of the $\mathrm{X}$ chromosome in $H$. lupulus may be due to a full-chromosome duplication followed by a fusion (see above), whereas the $\mathrm{Y}$ chromosome has remained unchanged. Assemblies of the H. lupulus sex chromosomes will be needed to test these hypotheses.

Our estimates of the age of the H. lupulus sex chromosomes are larger than the estimates for $C$. sativa, although we found very similar $\mathrm{X}-\mathrm{Y}$ maximum divergence in both species (higher bound age estimates are $c .50 \mathrm{Myr}$ and c. $28 \mathrm{Myr}$ old; highest $d S$ values 
are 0.362 and 0.415 in $H$. lupulus and C. sativa, respectively, see Prentout et al., 2020). Of course, the molecular clocks that we used are known to provide very rough estimates as they derive from the relatively distant Arabidopsis genus, and are sensitive to potential differences in mutation rates between the annual $C$. sativa and the perennial H. lupulus (Neve, 1991; Petit \& Hampe, 2006; Small, 2015; but see Krasovec et al., 2018). Indeed, only one of these molecular clocks (which is based on the mutation rate) takes into account the generation time ( $2 \mathrm{yr}$ in $H$. lupulus vs $1 \mathrm{yr}$ in C. sativa). This produced age estimate is approximately two-fold greater than that from the other clock (based on the substitution rate), which was not the case with C. sativa (Prentout et al., 2020). It is not known, however, if the generation time influences the substitution rate (Petit \& Hampe, 2006). Furthermore, the short generation time in C. sativa probably is a derived trait, not reflecting the long-term generation time of the Cannabis-Humulus lineage, as the Cannabis genus is the only herbaceous genus in the Cannabaceae family (Yang et al., 2013). Thus, the remarkable similarity between the highest $d S$ values in both species indicates that the $C$. sativa and H. lupulus sex chromosomes have a similar age, as expected if they derive from the same common ancestor. Although it is not possible to estimate their age exactly with the current data, initial recombination suppression at least pre-dates the split between the genera, that occurred between 21 and $25 \mathrm{Myr}$ ago (Ma) (Divashuk et al., 2014; Jin et al., 2020), and might even be $50 \mathrm{Myr}$ old. We thus confirmed here that the $\mathrm{XY}$ system shared by $C$. sativa and $H$. lupulus is among the oldest plant sex chromosome systems documented so far (Prentout et al., 2020).

Dioecy was inferred as the ancestral sexual system for the Cannabaceae, Urticaceae and Moraceae (Zhang et al., 2019; note, however, that many monoecious Cannabaceae were not included). We found that the synonymous divergence between the Cannabaceae species and Morus notabilis was approximately 0.45 , higher than the maximum divergence of the $\mathrm{X}$ and $\mathrm{Y}$ copies in the Cannabaceae. It remains possible that the sex chromosomes evolved before the split of the Cannabaceae and Moraceae families, because the oldest genes might have been lost or were not detected in our transcriptome data. There is, however, no report of whether or not sex chromosomes exist in Urticaceae and Moraceae (Ming et al., 2011).

In order to estimate the $\mathrm{Y}$ expression, we counted the number of reads with Y SNPs. Therefore, the impact of a potential Y reads mapping bias should be weaker on $Y$ expression analysis than on $\mathrm{X}-\mathrm{Y}$ divergence analysis. We validated this assumption by removing genes with detected mapping bias from the analysis, which did not change the signal of $\mathrm{Y}$ expression reduction and dosage compensation (Table S6; Figs S9, S10). Dosage compensation is a well-known phenomenon in animals (e.g. Gu \& Walters, 2017), but it has been documented only quite recently in plants (reviewed in Muyle et al., 2017). Here we found evidence for dosage compensation in $H$. lupulus; this is not surprising as previous work reported dosage compensation in C. sativa and we showed here that both systems are homologous. Cannabis sativa and $H$. lupulus add up to the list of plant sex chromosome systems with dosage compensation (see Muyle et al., 2017, for a review; and Prentout et al., 2020, and Fruchard et al., 2020, for the latest reports of dosage compensation in plants). Further analyses are needed to determine whether this dosage compensation has been selected or is an outcome of regulatory feedback (Malone et al., 2012; Krasovec et al., 2019).

Humulus lupulus sex chromosomes, like those of $C$. sativa, are well-differentiated, with a large nonrecombining region. Both species show similar patterns of $Y$ degeneration and dosage compensation, despite the fact that a large part of the nonrecombining region evolved independently in both species. These similarities, as well as the age of the chromosomes and the fact that they have been conserved since the most recent common ancestor of the two genera - a unique situation in plants so far provide an exciting opportunity to test and elaborate hypotheses on sex chromosome evolution in plants.

\section{Acknowledgements}

We thank Roberto Bacilieri for his help in setting up this collaboration and for discussions, Aline Muyle for advice on SEXDETECTOR and Florian Bénitière for helpful suggestions regarding graphical representations. This work was performed using the computing facilities of the CC LBBE/PRABI; we thank Bruno Spataro and Stéphane Delmotte for cluster maintenance. Virtual machines from the Institut Français de Bioinformatique also were used to perform this work. This work received financial support from grant no. P4-0077 from ARRS (Slovenian Research Agency) to JJ and from an ANR grant (ANR-14-CE19-0021-01) to GABM.

\section{Author contributions}

GABM, JK and DP conceptualized the study; GABM, JK, DP, NS and JJ formulated the methodology; DP, TT and CB-A operated the software; DP, TT and CB-A carried out the formal analysis; DP, NS, TT, CB-A, JJ, JK and GABM carried out the investigation; AC, NS and JJ supplied resources; DP, GABM, JK and TT wrote the original draft; all authors reviewed and edited the paper; DP and TT made the graphical representations; GABM and JK supervised the project; GABM administrated the project administration; and NS, JJ and GABM acquired funding. $\mathrm{JK}$ and GABM contributed equally to this work.

\section{ORCID}

Jernej Jakse (D) https://orcid.org/0000-0002-8907-1627

Jos Käfer (ID https://orcid.org/0000-0002-0561-8008

Gabriel A. B. Marais (D) https://orcid.org/0000-0003-2134-5967

Djivan Prentout (D) https://orcid.org/0000-0002-3088-3954

Theo Tricou (iD https://orcid.org/0000-0002-4432-2680

\section{Data availability}

The sequence data were deposited under the Bioproject with accession number PRJNA694508, BioSample SAMN17526021 (SRR13528971; SRR13528970; SRR13528969; SRR13528968; 
SRR13528966; SRR13528965; SRR13528964; SRR13528967; SRR13528963; SRR13528962; SRR13528961; SRR13528960; SRR13528959; SRR13528958).

\section{References}

Altschul SF, Gish W, Miller W, Myers EW, Lipman DJ. 1990. Basic local alignment search tool. Journal of Molecular Biology 215: 403-410.

Bačovský V, Čegan R, Šimoníková D, Hřibová E, Hobza R. 2020. The formation of sex chromosomes in Silene latifolia and S. dioica was accompanied by multiple chromosomal rearrangements. Frontiers Plant Science 11: 205.

Badouin H, Velt A, Gindraud F, Flutre T, Dumas V, Vautrin S, Marande W, Corbi J, Sallet E, Ganofsky J et al. 2020. The wild grape genome sequence provides insights into the transition from dioecy to hermaphroditism during grape domestication. Genome Biology 21: 1-24.

van Bakel H, Stout JM, Cote AG, Tallon CM, Sharpe AG, Hughes TR, Page JE. 2011. The draft genome and transcriptome of Cannabis sativa. Genome Biology 12: 1-18.

Baránková S, Pascual-Díaz JP, Sultana N, Alonso-Lifante MP, Balant M, Barros K, D'Ambrosio U, Malinská H, Peska V, Lorenzo IP et al. 2020. Sex-chrom, a database on plant sex chromosomes. New Phytologist 227: 1594-1604.

Barth-Haas GmbH \& Co. KG. 2019. Barth Report (1950-2019). Nuremberg. [WWW document] URL https://www.barthhaas.com/fileadmin/user_upload/ news/2019-07-23/barthreport20182019en.pdf. Accessed: 4 May 2020.

Castresana J. 2000. Selection of conserved blocks from multiple alignments for their use in phylogenetic analysis. Molecular Biology and Evolution 17: 540552.

Čerenak A, Kolenc Z, Sehur P, Whittock SP, Koutoulis A, Beatson R, Buck E, Javornik B, Škof S, Jakše J. 2019. New male specific markers for hop and application in breeding program. Scientific Reports 9: 1-9.

Charlesworth B, Charlesworth D. 2000. The degeneration of Y chromosomes. Philosophical Transactions of the Royal Society of London. Series B: Biological Sciences 355: 1563-1572.

Charlesworth D. 2016. Plant sex chromosomes. Annual Review of Plant Biology 67: 397-420.

Cherif E, Zehdi-Azouzi S, Crabos A, Castillo K, Chabrillange N, Pintaud J-C, Salhi-Hannachi A, Glémin S, Aberlenc-Bertossi F. 2016. Evolution of sex chromosomes prior to speciation in the dioecious Phoenix species. Journal of Evolutionary Biology 29: 1513-1522.

Conway S, Snyder R. 2008. Humulus lupulus - hops. College seminar 235 food for thought: the science, culture, \& politics of food. [WWW document] URL https:// academics.hamilton.edu/foodforthought/Our_Research_files/hops.pdf. [accessed 4 May 2020].

Cortez D, Marin R, Toledo-Flores D, Froidevaux L, Liechti A, Waters PD, Grützner F, Kaessmann H. 2014. Origins and functional evolution of $Y$ chromosomes across mammals. Nature 508: 488-493.

Divashuk MG, Alexandrov OS, Kroupin PY, Karlov GI. 2011. Molecular cytogenetic mapping of Humulus lupulus sex chromosomes. Cytogenetic and Genome Research 134: 213-219.

Divashuk MG, Alexandrov OS, Razumova OV, Kirov IV, Karlov GI. 2014. Molecular cytogenetic characterization of the dioecious Cannabis sativa with an XY chromosome sex determination system. PLoS ONE 9: e85118.

Dixon G, Kitano J, Kirkpatrick M. 2019. The origin of a new sex chromosome by introgression between two stickleback fishes. Molecular Biology and Evolution 36: 28-38.

Fridolfsson A-K, Cheng H, Copeland NG, Jenkins NA, Liu H-C, Raudsepp T, Woodage T, Chowdhary B, Halverson J, Ellegren H. 1998. Evolution of the avian sex chromosomes from an ancestral pair of autosomes. Proceedings of the National Academy of Sciences, USA 95: 8147-8152.

Fruchard C, Badouin H, Latrasse D, Devani RS, Muyle A, Rhoné B, Renner SS, Banerjee AK, Bendahmane A, Marais GAB. 2020. Evidence for dosage compensation in Coccinia grandis, a plant with a highly heteromorphic XY system. Genes 11: 787.

Gayral P, Melo-Ferreira J, Glémin S, Bierne N, Carneiro M, Nabholz B, Lourenco JM, Alves PC, Ballenghien M, Faivre N et al. 2013. Reference-free population genomics from next-generation transcriptome data and the vertebrate-invertebrate gap. PLoS Genetics 9: e1003457.

Grassa CJ, Wenger JP, Dabney C, Poplawski SG, Motley ST, Michael TP, Schwartz CJ, Weiblen GD. 2018. A complete Cannabis chromosome assembly and adaptive admixture for elevated cannabidiol (CBD) content. BioRxiv: 458083. doi: $10.1101 / 458083$.

Gu L, Walters JR. 2017. Evolution of sex chromosome dosage compensation in animals: a beautiful theory, undermined by facts and bedeviled by details ( $\mathrm{K}$ Makova, Ed.). Genome Biology and Evolution 9: 2461-2476.

Gu Z, Gu L, Eils R, Schlesner M, Brors B. 2014. circlize implements and enhances circular visualization in R. Bioinformatics 30: 2811-2812.

Harkess A, Zhou J, Xu C, Bowers JE, Van der Hulst R, Ayyampalayam S, Mercati F, Riccardi P, McKain MR, Kakrana A et al. 2017. The asparagus genome sheds light on the origin and evolution of a young Y chromosome. Nature Communications 8: 1-10.

He N, Zhang C, Qi X, Zhao S, Tao Y, Yang G, Lee T-H, Wang X, Cai Q, Li D et al. 2013. Draft genome sequence of the mulberry tree Morus notabilis. Nature Communications 4: 1-9.

Jakse J, Cerenak A, Radisek S, Satovic Z, Luthar Z, Javornik B. 2013. Identification of quantitative trait loci for resistance to Verticillium wilt and yield parameters in hop (Humulus lupulus L.). Theoretical and Applied Genetics 126: 1431-1443.

Jin J-J, Yang M-Q, Fritsch PW, van Velzen R, Li D-Z, Yi T-S. 2020. Born migrators: historical biogeography of the cosmopolitan family Cannabaceae. Journal of Systematics and Evolution 58: 461-473.

Käfer J, Marais GAB, Pannell JR. 2017. On the rarity of dioecy in flowering plants. Molecular Ecology 26: 1225-1241.

Kalyaanamoorthy S, Minh BQ, Wong TKF, von Haeseler A, Jermiin LS. 2017. ModelFinder: fast model selection for accurate phylogenetic estimates. Nature Methods 14: 587-589.

Karlov GI, Danilova TV, Horlemann C, Weber G. 2003. Molecular cytogenetics in hop (Humulus lupulus L.) and identification of sex chromosomes by DAPIbanding. Euphytica 132: 185-190.

Katsura Y, Iwase M, Satta Y. 2012. Evolution of genomic structures on mammalian sex chromosomes. Current Genomics 13: 115-123.

King M, Pavlovic M. 2017. Analysis of hop use in craft breweries in Slovenia. Journal of Agriculture Food and Development 3: 21-26.

Koch MA, Haubold B, Mitchell-Olds T. 2000. Comparative evolutionary analysis of chalcone synthase and alcohol dehydrogenase loci in Arabidopsis, Arabis, and related genera (Brassicaceae). Molecular Biology and Evolution 17: 1483-1498.

Kozlov AM, Darriba D, Flouri T, Morel B, Stamatakis A. 2019. RAxML-NG: a fast, scalable and user-friendly tool for maximum likelihood phylogenetic inference. Bioinformatics 35: 4453-4455.

Krasovec M, Chester M, Ridout K, Filatov DA. 2018. The mutation rate and the age of the sex chromosomes in Silene latifolia. Current Biology 28: 1832-1838.

Krasovec M, Kazama Y, Ishii K, Abe T, Filatov DA. 2019. Immediate dosage compensation is triggered by the deletion of y-linked genes in Silene latifolia. Current Biology 29: 2214-2221.

Krasovec M, Zhang Y, Filatov DA. 2020. The location of the pseudoautosomal boundary in Silene latifolia. Genes 11: 610.

Lartillot N, Lepage T, Blanquart S. 2009. PhyloBayes 3: a Bayesian software package for phylogenetic reconstruction and molecular dating. Bioinformatics 25: 2286-2288.

Lawson MJ, Zhang L. 2009. Sexy gene conversions: locating gene conversions on the X-chromosome. Nucleic Acids Research 37: 4570-4579.

Lemaitre C, Braga MDV, Gautier C, Sagot M-F, Tannier E, Marais GAB. 2009. Footprints of inversions at present and past pseudoautosomal boundaries in human sex chromosomes. Genome Biology and Evolution 1: 56-66.

Li H, Handsaker B, Wysoker A, Fennell T, Ruan J, Homer N, Marth G, Abecasis G, Durbin R, 1000 Genome Project Data Processing Subgroup. 2009. The sequence alignment/map format and SAMtools. Bioinformatics 25 : 2078-2079.

Mackinnon D, Pavlovič M. 2019. Global hop market analysis within the International Hop Growers' Convention. Hmeljarski Bilten 26: 99-108.

Malone JH, Cho D-Y, Mattiuzzo NR, Artieri CG, Jiang L, Dale RK, Smith HE, McDaniel J, Munro S, Salit M et al. 2012. Mediation of Drosophila 
autosomal dosage effects and compensation by network interactions. Genome Biology 13: 1-17.

Massonnet M, Cochetel N, Minio A, Vondras AM, Lin J, Muyle A, Garcia JF, Zhou Y, Delledonne M, Riaz S et al. 2020. The genetic basis of sex determination in grapes. Nature Communications 11: 1-12.

Ming R, Bendahmane A, Renner SS. 2011. Sex chromosomes in land plants. Annual Review of Plant Biology 62: 485-514.

Muyle A, Käfer J, Zemp N, Mousset S, Picard F, Marais GA. 2016. SEXDETECTOR: a probabilistic approach to study sex chromosomes in non-model organisms. Genome Biology and Evolution 8: 2530-2543.

Muyle A, Shearn R, Marais GA. 2017. The evolution of sex chromosomes and dosage compensation in plants. Genome Biology and Evolution 9: 627-645.

Muyle A, Zemp N, Deschamps C, Mousset S, Widmer A, Marais GAB. 2012. Rapid de novo evolution of $\mathrm{x}$ chromosome dosage compensation in Silene latifolia, a plant with young sex chromosomes. PLoS Biology 10: e1001308.

Neve RA. 1991. Hops. London, UK: Chapman and Hall.

Nguyen L-T, Schmidt HA, von Haeseler A, Minh BQ. 2015. IQ-TREE: a fast and effective stochastic algorithm for estimating maximum-likelihood phylogenies. Molecular Biology and Evolution 32: 268-274.

Nicolas M, Marais G, Hykelova V, Janousek B, Laporte V, Vyskot B, Mouchiroud D, Negrutiu I, Charlesworth D, Monéger F. 2004. A gradual process of recombination restriction in the evolutionary history of the sex chromosomes in dioecious plants. PLoS Biology 3: e4.

Ohno S. 1969. Evolution of sex chromosomes in mammals. Annual Review of Genetics 3: 495-524.

Okada Y, Ito K. 2001. Cloning and Analysis of Valerophenone Synthase Gene Expressed Specifically in Lupulin Gland of Hop (Humulus lupulus L.). Bioscience, Biotechnology \& Biochemistry 65: 150-155.

Ossowski S, Schneeberger K, Lucas-Lledó JI, Warthmann N, Clark RM, Shaw RG, Weigel D, Lynch M. 2010. The rate and molecular spectrum of spontaneous mutations in Arabidopsis thaliana. Science 327: 92-94.

Padgitt-Cobb LK, Kingan SB, Wells J, Elser J, Kronmiller B, Moore D, Concepcion G, Peluso P, Rank D, Jaiswal P. 2019. A phased, diploid assembly of the Cascade hop (Humulus lupulus) genome reveals patterns of selection and haplotype variation. BioRxiv: 786145. doi: 10.1101/786145.

Pattengale ND, Alipour M, Bininda-Emonds ORP, Moret BME, Stamatakis A. 2010. How many bootstrap replicates are necessary? Journal of Computational Biology 17: 337-354.

Patzak J, Nesvadba V, Chmelarsky I, Vejl P, Skupinová S. 2002. Identification of sex in F1 progenies of hop (Humulus lupulus) by molecular marker. Plant, Soil \& Environment 48: 318-321.

Peil A, Flachowsky H, Schumann E, Weber WE. 2003. Sex-linked AFLP markers indicate a pseudoautosomal region in hemp (Cannabis sativa L.). Theoretical and Applied Genetics 107: 102-109.

Peneder P, Wallner B, Vogl C. 2017. Exchange of genetic information between therian $\mathrm{X}$ and $\mathrm{Y}$ chromosome gametologs in old evolutionary strata. Ecology and Evolution 7: 8478-8487.

Petit RJ, Hampe A. 2006. Some evolutionary consequences of being a tree. Annual Review of Ecology, Evolution \& Systematics 37: 187-214.

Prentout D, Razumova O, Rhoné B, Badouin H, Henri H, Feng C, Käfer J, Karlov G, Marais GAB. 2020. An efficient RNA-seq-based segregation analysis identifies the sex chromosomes of Cannabis sativa. Genome Research 30: 164172.

Polley A, Ganal MW, Seigner E. 1997. Identification of sex in hop (Humulus lupulus) using molecular markers. Genome 40: 357-361.

Pucholt P, Wright AE, Conze LL, Mank JE, Berlin S. 2017. Recent sex chromosome divergence despite ancient dioecy in the Willow Salix viminalis. Molecular Biology and Evolution 34: 1991-2001.

Quinlan AR, Hall IM. 2010. BEDTools: a flexible suite of utilities for comparing genomic features. Bioinformatics 26: 841-842.

R Core Team. 2018. R: A language and environment for statistical computing, v.3.4.4. Vienna, Austria: R Foundation for Statistical Computing. [WWW document] URL https://www.R-project.org/.

Ranwez V, Harispe S, Delsuc F, Douzery EJP. 2011. MACSE: multiple alignment of coding SEquences accounting for frameshifts and stop codons. PLoS ONE 6: e22594.
Raymond O, Gouzy J, Just J, Badouin H, Verdenaud M, Lemainque A, Vergne P, Moja S, Choisne N, Pont C et al. 2018. The Rosa genome provides new insights into the domestication of modern roses. Nature Genetics 50: 772-777.

Renner SS. 2014. The relative and absolute frequencies of angiosperm sexual systems: dioecy, monoecy, gynodioecy, and an updated online database. American Journal of Botany 101: 1588-1596.

Renner SS, Müller NA. 2021. Plant sex chromosomes defy evolutionary models of expanding recombination suppression and genetic degeneration. Nature Plants 1-11.

Ross MT, Grafham DV, Coffey AJ, Scherer S, McLay K, Muzny D, Platzer M, Howell GR, Burrows C, Bird CP et al. 2005. The DNA sequence of the human X chromosome. Nature 434: 325-337.

Sawyer S. 1999. GENECONV: A computer package for the statistical detection of gene conversion. [WWW document] URL http://www.math.wustl.edu/-sawyer.

Shephard HL, Parker JS, Darby P, Ainsworth CC. 2000. Sexual development and sex chromosomes in hop. New Phytologist 148: 397-411.

Shulaev V, Sargent DJ, Crowhurst RN, Mockler TC, Folkerts O, Delcher AL, Jaiswal P, Mockaitis K, Liston A, Mane SP et al. 2011. The genome of woodland strawberry (Fragaria vesca). Nature Genetics 43: 109-116.

Skaletsky H, Kuroda-Kawaguchi T, Minx PJ, Cordum HS, Hillier LD, Brown LG, Repping S, Pyntikova T, Ali J, Bieri T et al. 2003. The male-specific region of the human $\mathrm{Y}$ chromosome is a mosaic of discrete sequence classes. Nature 423: 825-837.

Small E. 2015. Evolution and classification of Cannabis sativa (Marijuana, Hemp) in relation to human utilization. The Botanical Review 81: 189-294.

Sousa A, Fuchs J, Renner SS. 2013. Molecular cytogenetics (FISH, GISH) of Coccinia grandis: A ca. 3 myr-old species of cucurbitaceae with the largest $\mathrm{Y} /$ autosome divergence in flowering plants. Cytogenetic and Genome Research 139: $107-118$.

Takahata N, Nei M. 1985. Gene genealogy and variance of interpopulational nucleotide differences. Genetics 110: 325-344.

Thomas GG, Neve RA. 1976. Studies on the effect of pollination on the yield and resin content of hops (Humulus lupulus L.). Journal of the Institute of Brewing 82: 41-45.

Torres MF, Mathew LS, Ahmed I, Al-Azwani IK, Krueger R, Rivera-Nuñez D, Mohamoud YA, Clark AG, Suhre K, Malek JA. 2018. Genus-wide sequencing supports a two-locus model for sex-determination in Phoenix. Nature Communications 9: 1-9.

Trombetta B, Sellitto D, Scozzari R, Cruciani F. 2014. Inter- and intraspecies phylogenetic analyses reveal extensive X-Y gene conversion in the evolution of gametologous sequences of human sex chromosomes. Molecular Biology and Evolution 31: 2108-2123.

van Velzen R, Holmer R, Bu F, Rutten L, van Zeijl A, Liu W, Santuari L, Cao $\mathrm{Q}$, Sharma T, Shen D et al. 2018. Comparative genomics of the nonlegume Parasponia reveals insights into evolution of nitrogen-fixing rhizobium symbioses. Proceedings of the National Academy of Sciences, USA 115: E4700E4709.

Wang J, Na J-k, Yu Q, Gschwend Ar, Han J, Zeng F, Aryal R, VanBuren R, Murray Je, Zhang W et al. 2012. Sequencing papaya X and Yh chromosomes reveals molecular basis of incipient sex chromosome evolution. Proceedings of the National Academy of Sciences, USA 109: 13710-13715.

Westergaard M. 1958. The mechanism of sex determination in dioecious flowering plants. Advances in Genetics 9: 217-281.

Wickham H. 2011. ggplot2. Wiley Interdisciplinary Reviews: Computational Statistics 3: 180-185.

Wright AE, Dean R, Zimmer F, Mank JE. 2016. How to make a sex chromosome. Nature Communications 7: 1-8.

Wu M, Moore RC. 2015. The evolutionary tempo of sex chromosome degradation in Carica papaya. Journal of Molecular Evolution 80: 265-277.

Wu TD, Nacu S. 2010. Fast and SNP-tolerant detection of complex variants and splicing in short reads. Bioinformatics 26: 873-881.

Wu TD, Reeder J, Lawrence M, Becker G, Brauer MJ. 2016. GMAP and GSNAP for genomic sequence alignment: enhancements to speed, accuracy, and functionality. Methods in Molecular Biology 1418: 283-334.

Yang M-Q, van Velzen R, Bakker FT, Sattarian A, Li D-Z, Yi T-S. 2013. Molecular phylogenetics and character evolution of Cannabaceae. Taxon 62: 473-485. 
Yang Z. 2007. PAML 4: phylogenetic analysis by maximum likelihood. Molecular Biology and Evolution 24: 1586-1591.

Zhang Q, Onstein RE, Little SA, Sauquet H. 2019. Estimating divergence times and ancestral breeding systems in Ficus and Moraceae. Annals of Botany 123: 191-204.

\section{Supporting Information}

Additional Supporting Information may be found online in the Supporting Information section at the end of the article.

Fig. S1 Example of genes whose topology changed with the mapping bias filtering.

Fig. S2 Histogram of $(d S)$ between $C$. sativa and $M$. notabilis.

Fig. S3 Histogram of $d S$ between $C$. sativa and $R$. chinensis.

Fig. S4 Histogram of the Y/X expression ratio.

Fig. S5 Histogram of the Allele-specific expression analysis for the parents.

Fig. S6 Histogram of the Allele-specific expression analysis for the daughters in the nonrecombining region.

Fig. S7 Histogram of the Allele-specific expression analysis for the daughters out of the nonrecombining region.

Fig. S8 SEX-DETector inference errors due to WGD in H. lupulus.

Fig. S9 Y/X expression ratio along the sex chromosome without genes with a detected mapping bias.
Fig. S10 Dosage compensation analysis without genes with a detected mapping bias.

Methods S1 Methods for the RNA-seq mapping on $H$. lupulus reference, for the estimation of the synonymous divergence with two outgroup species and for the Allele-specific expression analysis.

Table S1 Summary of chromosome names in Fig. 2(a) and in assembly fasta file.

Table S2 Statistics of mapping on H. lupulus and C. sativa references.

Table S3 Summary of SEX-DETECTOR genotyping errors and inferences.

Table S4 SEX-DETECTOR assignment file output.

Table S5 Age estimates of the youngest strata in $H$. lupulus and C. sativa.

Table S6 Expression analysis statistics summary.

Please note: Wiley Blackwell are not responsible for the content or functionality of any Supporting Information supplied by the authors. Any queries (other than missing material) should be directed to the New Phytologist Central Office. 